\title{
Visual Hallucinations and Amyloid Deposition in Parkinson's Disease Dementia: A Case Report
}

\author{
Yoo Hyun Um¹, Tae-Won Kim¹, Jong-Hyun Jeong ${ }^{1}$, Ho-Jun Seo', Jin-Hee Han', Seung-Chul Hong', \\ Won-Sang Jung ${ }^{2}$, Woo Hee Choi ${ }^{3}$, Chang-Uk Lee ${ }^{4}$, and Hyun Kook Lim ${ }^{1} \bowtie$ \\ ${ }^{1}$ Department of Psychiatry, St. Vincent's Hospital, College of Medicine, The Catholic University of Korea, Suwon, Republic of Korea \\ 2Department of Radiology, St. Vincent's Hospital, College of Medicine, The Catholic University of Korea, Suwon, Republic of Korea \\ ${ }^{3}$ Department of Nuclear Medicine, St. Vincent's Hospital, College of Medicine, The Catholic University of Korea, Suwon, Republic of Korea \\ ${ }^{4}$ Department of Psychiatry, Seoul St. Mary's Hospital, College of Medicine, The Catholic University of Korea, Seoul, Republic of Korea
}

Parkinson's disease dementia (PDD) is notorious for its debilitating clinical course and high mortality rates. Consequently, various attempts to investigate predictors of cognitive decline in Parkinson's disease (PD) have been made. Here we report a case of a 75-year-old female patient with PD who visited the clinic with complaints of recurrent visual hallucinations and cognitive decline, whose symptoms were ameliorated by the titration of rivastigmine. Imaging results showed pronounced diffuse cortical amyloid deposition evidenced by 18F-florbetaben amyloid positron emission tomography (PET) imaging. This observation suggests that pronounced amyloid deposition and visual hallucinations in PD patients could be clinically significant predictors of cognitive decline in PD patients. Future research should concentrate on accumulating more evidence for possible predictors of cognitive decline and their association with PD pathology that can enable an early intervention and standardized treatment in PDD patients.

Psychiatry Investig 2016;13(3):364-369

Key Words Parkinson's disease, Dementia, Amyloid imaging, Cognitive decline.

\section{INTRODUCTION}

Parkinson's disease (PD) has long been well known for its association with progressive cognitive decline, with one third of them eventually diagnosed with dementia. ${ }^{1}$ Mortality rates of PD patients with dementia are significantly higher than those without dementia, with 3 years shorter life expectancy when compared with those without dementia. ${ }^{2}$ Therefore, early detection of cognitive decline with timely intervention has become of great importance for patients and clinicians.

Extant studies have adopted various modalities to find predictors of Parkinson's disease dementia (PDD). Two large-scale cohort studies have indicated that akinetic type of $\mathrm{PD}$, hallucinations, increasing age, deficits in semantic fluency and failure

Received: August 6, 2015 Revised: September 28, 2015 Accepted: September 28, 2015 Available online: February 17, 2016

$\triangle$ Correspondence: Hyun Kook Lim, MD, PhD

Department of Psychiatry, St. Vincent's Hospital, College of Medicine, The Catholic University of Korea, 93 Jungbu-daero, Paldal-gu, Suwon 16247, Republic of Korea

Tel: +82-31-249-8167, Fax: +82-31-248-6758, E-mail: drblues@catholic.ac.kr

(c) This is an Open Access article distributed under the terms of the Creative Commons Attribution Non-Commercial License (http://creativecommons.org/licenses/by$\mathrm{nc} / 3.0$ ) which permits unrestricted non-commercial use, distribution, and reproduction in any medium, provided the original work is properly cited. to draw intersected pentagons have significant association with the risk of future PDD development. ${ }^{3,4}$ Motor symptom severity and apathy have also been reported to be able to predict cognitive decline in PD patients. ${ }^{4,5}$ In neuroimaging studies, a close association between Alzheimer's disease (AD) pathology demonstrated by magnetic resonance imaging (MRI) and deficits in cognitive performances of PD has been delineated. ${ }^{6}$ Moreover, cortical amyloid burden has been purported to be able to predict the rate of evolving into dementia in PD patients. ${ }^{7}$ Genetic studies have indicated the possibility of increased risk of dementia in Apolipoprotein $\varepsilon 2$ allele carriers and microtubule-associated protein tau (MAPT) genotype carriers. $^{4,8}$

Relatively scarce studies have been performed to demonstrate marked amyloid deposition in PDD patients utilizing invivo amyloid imaging. Here, we present a case of PD patient with visual hallucinations and significant amyloid burden evidenced by beta-amyloid imaging, with pronounced cognitive impairment demonstrated by neuropsychological tests. There is a paucity of evidence on the clinical application of beta-amyloid imaging in PD patients, and the present case report will add another dimension to imaging diagnosis of cognitive decline in PD. In addition, a retrospective review of clinical course 
and diagnostic processes in this patient will raise questions on possible predictors of cognitive decline in PD. As with treatment approaches, several recent suggestions on the management of visual hallucinations in PD patients will be discussed.

\section{CASE}

A 75-year-old female patient visited our clinic with complaints of recurrent visual hallucinations of animals and human figures. She had been diagnosed with PD for 5 years with bradykinesia and both hand tremors as incipient symptoms in a neurosurgical clinic. Other medical diagnoses in her past history included hypertension, hyperlipidemia and osteoporosis. She had been taking medications including levodopa/carbidopa/entacapone 150/37.5/200 mg twice a day, and $2 \mathrm{mg}$ of ropinirole daily to control parkinsonian symptoms. She started to complain recurrent visual hallucination 4 years after the diagnosis of PD. Those visual hallucinations were vivid while disturbing, evoking anxiety and often sleep disturbance in the patient. $12.5 \mathrm{mg}$ of quetiapine at night was prescribed to ameliorate the hallucinations but to no avail. Considering the degree of anxiety and the potential behavioral consequences of visual hallucinations she was experiencing, she was admitted to a psychiatric ward for more proper diagnosis and management.

Upon her psychiatric admission, neurological examination revealed masked face without tremors, moderate rigidity, bradykinesia, stooped posture, decreased arm swings and narrowbased gait with short steps. A neuropsychological test battery was used to test the patient's cognitive status. She scored 16 in Mini-mental status examination, 1 in Clinical dementia rating scale (CDR), and 3.5 in Clinical Dementia Rating-Sum of Box score (CDR-SB). ${ }^{9,10}$ In addition, she scored 25 in Neuropsychiatric Inventory (NPI), ${ }^{11}$ with major scores centered on symptoms of hallucination, depression, anxiety and apathy. Results from the Seoul-Instrumental activity of daily living (S-IADL) indicated score of $8,{ }^{12}$ which indicated that the patient had been experiencing mild impairments in instrumental activity of daily living. A Korean version of the Consortium to Establish a Registry for Alzheimer's Disease Assessment Packet (CERAD-K) was used to assess the patient's cognitive function. ${ }^{13,14}$ Results revealed marked impairments in Korean Boston naming test, word recognition and recall tests as well as constructional recall test, all falling below 5thpercentile scores ${ }^{13}$ (Table 1).

Brain MRI revealed marked medial temporal lobe atrophy (grade 2 by medial temporal lobe atrophy visual rating scale) with global cerebral atrophy (grade 1 by cortical atrophy scale) and periventricular, deep white matter hyperintensities (grade 2 by Fazeka scale) ${ }^{15-17}$ (Figure 1). A fluorodopa F18 positron emission tomography (PET) was implemented to confirm the diagnosis of PD in the midbrain after discontinuing dopaminergic agents for three days. Decreased activity of both posterior portions of putamina was noted, and mild decrements in both caudate activities were found (Figure 2), concordant with the PD pathology. Patterns of the patient's cognitive decline fulfilled the National Institute of Neurological and Communicative Disorders and Stroke and the Alzheimer's Disease and Related Disorders Association Alzheimer's (NINCDS-ADRDA) criteria $^{18}$ for possible Alzheimer's disease while mixed etiologies, making the patient a good candidate for amyloid PET imaging. ${ }^{19}$ Amyloid imaging with $18 \mathrm{~F}$-florbetaben was performed to acquire images of cortical diffusion in the patient (Figure 2). Brain beta-amyloid plaque load (BAPL) was found to have a

Table 1. Demographic characteristics and CERAD-K results of the patient

\begin{tabular}{lcc}
\hline Demographic characteristics & & \\
\hline Age & 75 & \\
Sex & Female & \\
Educational level & 2 years & 6.0 \\
\hline CERAD-K results & & 3.0 \\
\hline Verbal fluency & 7 & 14.9 \\
Korean boston naming test & 2 & 6.0 \\
MMSE-KC & 16 & 2.0 \\
Word list memory & 5 & 2.0 \\
Constructional praxis & 5 & 5.5 \\
Word list recall & 0 & 2.0 \\
Word list recognition & 1 &
\end{tabular}

CERAD-K: Korean version of the Consortium to Establish a Registry for Alzheimer's Disease Assessment Packet, MMSE-KC: the MiniMental State Examination in the Korean version 
score of 3 , indicating pronounced amyloid load. ${ }^{20}$

To control her visual hallucinations, increased doses up to $100 \mathrm{mg}$ of quetiapine was applied, and antiparkinsonian drugs (levodopa/carbidopa/entacapone 150/37.5/200 mg and $2 \mathrm{mg}$ of ropinirole) were discontinued to exclude the effect of dopaminergic agents on visual hallucination. However, there was no improvement in her symptoms. Significant distress due to oversedation as side effect of quetiapine was found. Her parkinsonian symptoms started to emerge after discontinuing those drugs, including persistent rigidity and bradykinesia resulting in mobility limitations. Alternative prescription was considered for her medication regimen. Her previous antiparkinsonian medications were discontinued, and instead, 100 $\mathrm{mg}$ of levodopa and $25 \mathrm{mg}$ of carbidopa were prescribed. The patient was required to take 1.5 tablets 3 times a day. In addition, $6 \mathrm{mg}$ of paliperidone was used, which was soon discontinued due to marked aggravation in rigidity and postural instability. To target her progressive memory decline, $9 \mathrm{mg}$ of rivastigmine patch was prescribed, and the patient experienced reduction in frequency of visual hallucinations. As a result, her anxiety subsided, and the dose of rivastigmine was increased up to $18 \mathrm{mg}$ upon her discharge after one month of
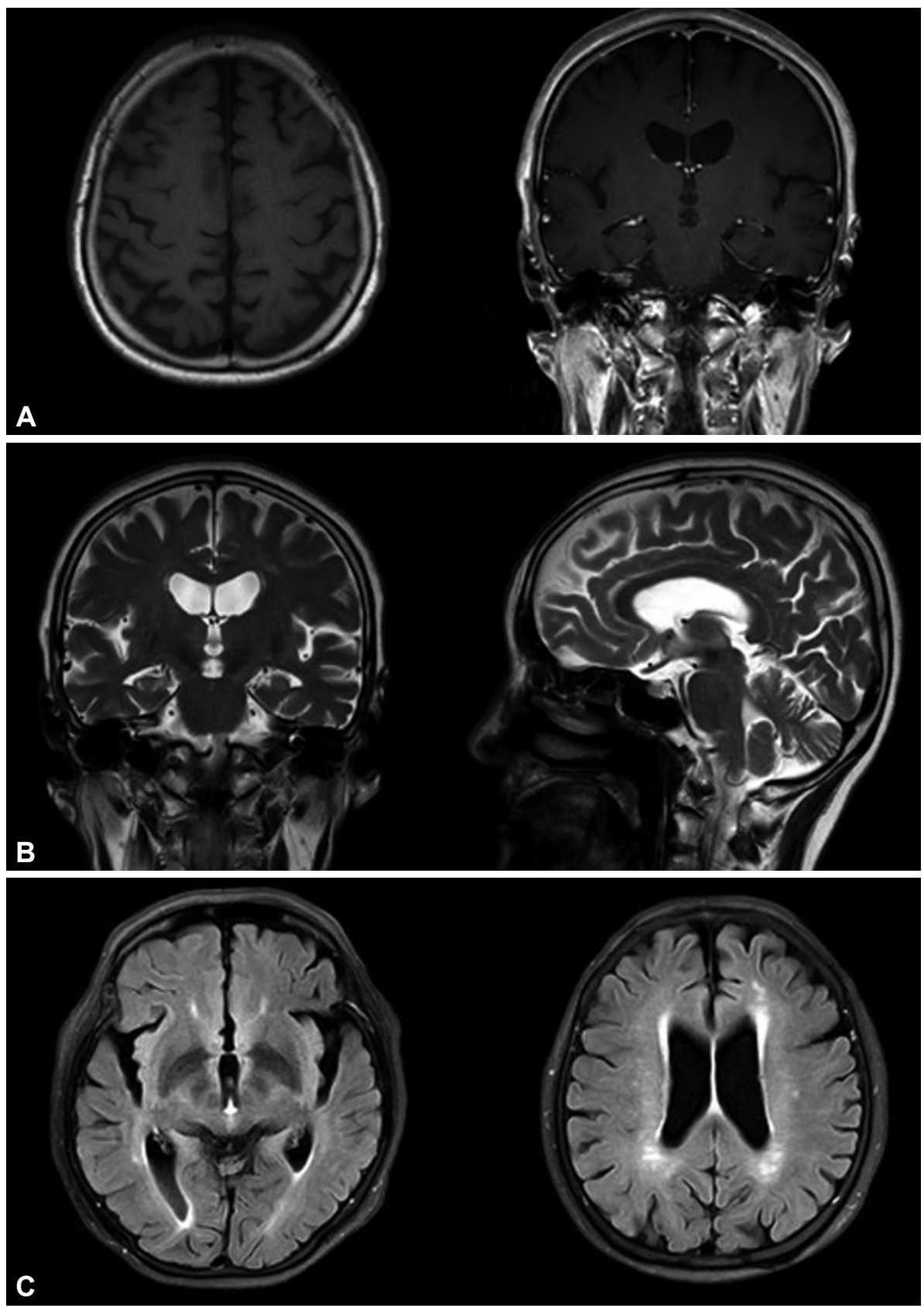

Figure 1. Brain magnetic resonance imaging of the patient. T1-weighted axial and coronal images (A), T2-weighted coronal and sagittal images (B), and fluid attenuated inversion recovery (FLAIR) axial images (C). 

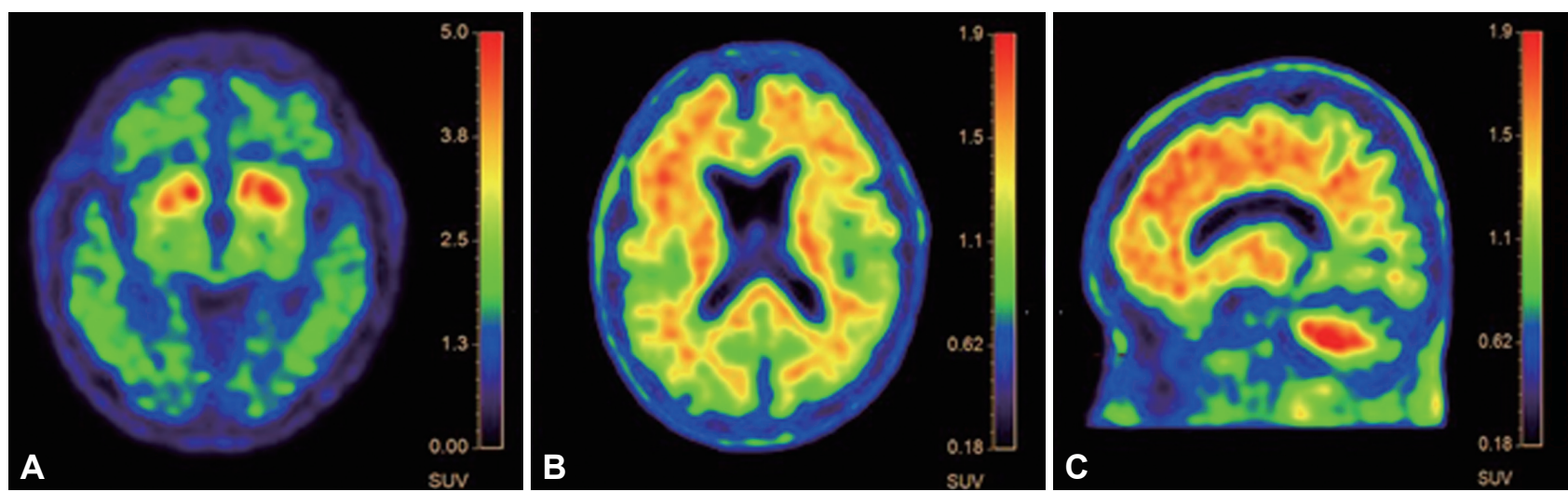

Figure 2. An axial image from Brain fluorodopa $F 18$ positron emission tomography (PET) $(A)$ and axial and sagittal images from amyloid imaging with $18 \mathrm{~F}-$ florbetaben (B and $\mathrm{C})$.

psychiatric admission.

\section{DISCUSSION}

Previous literatures have repeatedly reported the clinical and pathological overlaps between $\mathrm{AD}$ and $\mathrm{PD}$ pathologies. Some studies have demonstrated that the two neurodegenerative diseases share etiological basis. Linkage between presenillin 1 and alpha-synuclein has been elucidated in one study, ${ }^{21}$ whereas genetic polymorphisms and disruptions in iron homeostasis have been suggested as possible culprits. ${ }^{22}$ Oxidative damage amplified by mitochondrial derangement has been purported to be possible cause of amyloidogenesis and neuronal damage in both $\mathrm{AD}$ and $\mathrm{PD} .^{22}$ In addition, neuronal losses in locus coeruleus and disabled modulatory role of nicotinic receptors could also explain the shared clinical pictures of $\mathrm{AD}$ and $\mathrm{PD}^{22}$ Although the aforementioned overlaps complicated the diagnostic process, pondering over the etiological basis for the cognitive decline evident in the patient, we focused on the disparities in the cognitive impairment patterns of $\mathrm{AD}$ and PDD. Patients with PDD and dementia with Lewy bodies (DLB) tend to display more severe impairments in visual perception, recognition, constructional recall as well as in attention and fronto-executive function when compared with $\mathrm{AD}$ patients. ${ }^{23}$ Meanwhile, $\mathrm{AD}, \mathrm{PDD}$ and $\mathrm{DLB}$ patients can equally show disabilities in semantic memory. ${ }^{23}$ The cognitive profile of our patient demonstrated marked impairments in semantic memory, word recognition, and constructional recall test, which were concordant with the cognitive characteristics of PDD and DLB. The patient had been suffering from Parkinsonian symptoms long before the emergence of cognitive decline, which made our conclusion geared towards the diagnosis of PDD.

As with substantial $\mathrm{AD}$ pattern of cortical atrophy and high cortical amyloid burden evident in the patient, they offer some important and valuable clinical implications. A previous study has suggested that cortical atrophy resembling $\mathrm{AD}$ could be a valuable predictor of cognitive decline in cognitively normal PD patients. ${ }^{6}$ Significant cortical and hippocampal atrophy in the patient might represent risks of PDD that the patient had harbored before conversion to dementia. Mixed reports exist on the understanding of the role of cortical amyloid deposition. While infrequent amyloid deposition was noted in PDD patients when compared with DLB patients in one study, ${ }^{24}$ several literatures have consistently reported associations between cognitive decline and cerebral amyloid retention in PD without dementia. ${ }^{25-27}$ The marked cognitive impairment in this present case might have been foretold long before the manifestation of actual symptoms.

Meanwhile, visual hallucinations in PDD patients have been interpreted from multidimensional aspects. Presence of hallucinations can expedite the rate of cognitive deterioration in PD patients. ${ }^{28}$ An approximately $20 \%$ of $\mathrm{PD}$ patients have been reported to have visual hallucinations, with marked cognitive impairment and longer duration of illness as predictors. ${ }^{29} \mathrm{Hal}-$ lucinations not only could predict the rates of cognitive decline, but also could predict the severity of cognitive impairment. ${ }^{30}$ Along with the bidirectional relationship between visual hallucination and cognitive decline in PD patient, a causal relationship between the two clinical entities has been delineated. Disruptions in networks involved in attentional control, ${ }^{31}$ along with perceptual disturbances originating from faulty processing of visual information are thought to be important causative factors of visual hallucinations in $\mathrm{PD}^{23} \mathrm{Im}$ pairments in detailed recollection of events have also been purported to be contributors to the emergence of visual hallucinations. ${ }^{32}$ Attempts have been made to integrate various suggested causes of visual hallucination into theoretical models with regard to anatomical correlates. ${ }^{33}$

Visual hallucinations from dopaminergic agents in PD have been effectively controlled by quetiapine in several studies, but our patient was not responsive to quetiapine. ${ }^{34-36}$ Some mixed results have been reported on the antipsychotics treatment of 
visual hallucination in $\mathrm{PD}$ patients. ${ }^{37}$ As with antidepressants, two recent case reports mirtazapine improving visual or auditory hallucinations have been notable, without side effects of aggravation in patients' motor symptoms. ${ }^{38,39}$ Non-pharmacological interventions yielded varying results. Case series enlisting application of electroconvulsive therapy (ECT) in PD patients with treatment-resistant psychotic symptoms have been delineated, and use of ECT in this group of patient could be worthy of attempt. ${ }^{40}$ With reports of low frequency transcranial magnetic stimulation (TMS) suppressing the activity of occipital cortex, ${ }^{41,42}$ application of TMS sounds feasible, but more evidences are needed.

Regarding the treatment approaches of visual hallucinations in this case, rivastigmine has been shown to be effective in controlling both cognitive symptoms and visual hallucinations in dementia associated with Parkinson's disease. ${ }^{43,44}$ The patient in this case also demonstrated amelioration of symptoms with the titration of rivastigmine. Previous studies ${ }^{45,46}$ have demonstrated the efficacy of anticholinesterase inhibitors as a whole in abating visual hallucinations and other neuropsychiatric symptoms associated with dementia. They have suggested that the effect of acetylcholinesterase inhibitors on visual hallucination of PDD patients might be due to increases in cholinergic neurotransmission resulting in improvements in attention and visual information processing, in accordance with the aforementioned discussion on the etiopathogenesis of visual hallucinations in PD patients. ${ }^{45,46}$

Further studies will be needed to find predictors of cognitive decline and their association with PD pathology. More clinical trials are required to establish a more standardized treatment approach for visual hallucinations and cognitive dysfunction frequently present in PDD patients.

\section{Acknowledgments}

This research was supported by Basic Science Research Program through the National Research Foundation of Korea(NRF) funded by the Ministry of Science, ICT \& Future Planning (NRF-2015R1C1A1A02036578).

\section{REFERENCES}

1. Aarsland D, Zaccai J, Brayne C. A systematic review of prevalence studies of dementia in Parkinson's disease. Mov Disord 2005;20:1255-1263.

2. Buter T, van den Hout A, Matthews F, Larsen J, Brayne C, Aarsland D. Dementia and survival in Parkinson disease: a 12-year population study. Neurology 2008;70:1017-1022.

3. Aarsland D, Andersen K, Larsen JP, Lolk A. Prevalence and characteristics of dementia in Parkinson disease: an 8-year prospective study. Arch Neurol 2003;60:387-392.

4. Williams-Gray CH, Evans JR, Goris A, Foltynie T, Ban M, Robbins TW, et al. The distinct cognitive syndromes of Parkinson's disease: 5 year follow-up of the CamPaIGN cohort. Brain 2009;132:2958-2969.

5. Fitts W, Weintraub D, Massimo L, Chahine L, Chen-Plotkin A, Duda JE, et al. Caregiver report of apathy predicts dementia in Parkinson's disease. Parkinsonism Relat Disord 2015;21:992-995.

6. Weintraub D, Dietz N, Duda JE, Wolk DA, Doshi J, Xie SX, et al. Al- zheimer's disease pattern of brain atrophy predicts cognitive decline in Parkinson's disease. Brain 2012;135:170-180.

7. Compta Y, Parkkinen L, O'Sullivan SS, Vandrovcova J, Holton JL, Collins C, et al. Lewy-and Alzheimer-type pathologies in Parkinson's disease dementia: which is more important? Brain 2011;134:1493-1505.

8. de Lau LM, Schipper CM, Hofman A, Koudstaal PJ, Breteler MM. Prognosis of parkinson disease: risk of dementia and mortality: the Rotterdam study. Arch Neurol 2005;62:1265-1269.

9. Hughes CP, Berg L, Danziger WL, Coben LA, Martin RL. A new clinical scale for the staging of dementia. Br J Psychiatry 1982;140:566-572.

10. O'Bryant SE, Waring SC, Cullum C, Hall J, Lacritz L, Massman PJ, et al. Staging dementia using clinical dementia rating scale sum of boxes scores: a Texas Alzheimer's research consortium study. Arch Neurol 2008;65:1091-1095.

11. Cummings JL, Mega M, Gray K, Rosenberg-Thompson S, Carusi DA, Gornbein J. The neuropsychiatric inventory: comprehensive assessment of psychopathology in dementia. Neurology 1994;44:2308-2314.

12. Ku HM, Kim JH, Kwon EJ, Kim SH, Lee HS, Ko HJ, et al. A study on the reliability and validity of Seoul-Instrumental Activities of Daily Living (S-IADL). J Korean Neuropsychiatr Assoc 2004;43:189-199.

13. Lee DY, Lee KU, Lee JH, Kim KW, Jhoo JH, Kim SY, et al. A normative study of the CERAD neuropsychological assessment battery in the Korean elderly. J Int Neuropsychol Soc 2004;10:72-81.

14. Lee JH, Lee KU, Lee DY, Jhoo JH, Kim KW, Woo JI. Korean version of the consortium to establish a registry for Alzheimer's disease assessment packet (CERAD-K): Clinical and neuropsychological assessment batteries. Neurobiol Aging 2000;21:32.

15. Scheltens P, Leys D, Barkhof F, Huglo D, Weinstein H, Vermersch P, et al. Atrophy of medial temporal lobes on MRI in "probable" Alzheimer's disease and normal ageing: diagnostic value and neuropsychological correlates. J Neurol Neurosurg Psychiatry 1992;55:967-972.

16. Wahlund L, Barkhof F, Fazekas F, Bronge L, Augustin M, Sjögren M, et al. A new rating scale for age-related white matter changes applicable to MRI and CT. Stroke 2001;32:1318-1322.

17. Scheltens P, Pasquier F, Weerts JG, Barkhof F, Leys D. Qualitative assessment of cerebral atrophy on MRI: inter-and intra-observer reproducibility in dementia and normal aging. Eur Neurol 1997;37:95-99.

18. McKhann G, Drachman D, Folstein M, Katzman R, Price D, Stadlan EM. Clinical diagnosis of Alzheimer's disease: report of the NINCDSADRDA Work Group under the auspices of Department of Health and Human Services Task Force on Alzheimer's Disease. Neurology 1984;34:939-944.

19. Johnson KA, Minoshima S, Bohnen NI, Donohoe KJ, Foster NL, Herscovitch P, et al. Appropriate use criteria for amyloid PET: a report of the Amyloid Imaging Task Force, the Society of Nuclear Medicine and Molecular Imaging, and the Alzheimer's Association. J Nucl Med 2013;54:476-490.

20. Sabri O, Seibyl J, Rowe C, Barthel H. Beta-amyloid imaging with florbetaben. Clin Transl Imaging 2015;3:13-26.

21. Winslow AR, Moussaud S, Zhu L, Post KL, Dickson DW, Berezovska $\mathrm{O}$, et al. Convergence of pathology in dementia with Lewy bodies and Alzheimer's disease: a role for the novel interaction of alpha-synuclein and presenilin 1 in disease. Brain 2014;137:1958-1970.

22. Xie A, Gao J, Xu L, Meng D. Shared mechanisms of neurodegeneration in Alzheimer's disease and Parkinson's disease. Biomed Res Int 2014; 2014:648740.

23. Metzler-Baddeley C. A review of cognitive impairments in dementia with Lewy bodies relative to Alzheimer's disease and Parkinson's disease with dementia. Cortex 2007;43:583-600.

24. Edison P, Rowe CC, Rinne JO, Ng S, Ahmed I, Kemppainen N, et al. Amyloid load in Parkinson's disease dementia and Lewy body dementia measured with [11C] PIB positron emission tomography. J Neurol Neurosurg Psychiatry 2008;79:1331-1338.

25. Gomperts SN, Locascio JJ, Rentz D, Santarlasci A, Marquie M, Johnson KA, et al. Amyloid is linked to cognitive decline in patients with Par- 
kinson disease without dementia. Neurology 2013;80:85-91.

26. Svenningsson P, Westman E, Ballard C, Aarsland D. Cognitive impairment in patients with Parkinson's disease: diagnosis, biomarkers, and treatment. Lancet Neurol 2012;11:697-707.

27. Compta Y, Parkkinen L, Kempster P, Selikhova M, Lashley T, Holton JL, et al. The significance of $\alpha$-synuclein, amyloid $-\beta$ and tau pathologies in Parkinson's disease progression and related dementia. Neurodegener Dis 2014;13:154-156.

28. Aarsland D, Andersen K, Larsen JP, Perry R, Wentzel-Larsen T, Lolk A, et al. The rate of cognitive decline in Parkinson disease. Arch Neurol 2004;61:1906-1911.

29. Fénelon G, Mahieux F, Huon R, Ziégler M. Hallucinations in Parkinson's disease. Prevalence, phenomenology, and risk factors. Brain 2000; 123:733-745.

30. Factor SA, Scullin MK, Sollinger AB, Land JO, Wood-Siverio C, Zanders L, et al. Cognitive correlates of hallucinations and delusions in Parkinson's disease. J Neurol Sci 2014;347:316-321.

31. Shine JM, Halliday GM, Gilat M, Matar E, Bolitho SJ, Carlos M, et al. The role of dysfunctional attentional control networks in visual misperceptions in Parkinson's disease. Human Brain Mapp 2014;35:22062219.

32. Barnes J, Boubert L, Harris J, Lee A, David AS. Reality monitoring and visual hallucinations in Parkinson's disease. Neuropsychologia 2003; 41:565-574.

33. Muller AJ, Shine JM, Halliday GM, Lewis SJ. Visual hallucinations in Parkinson's disease: theoretical models. Mov Disord 2014;29:15911598.

34. Ondo WG, Tintner R, Voung KD, Lai D, Ringholz G. Double-blind, placebo-controlled, unforced titration parallel trial of quetiapine for dopaminergic-induced hallucinations in Parkinson's disease. Mov Disord 2005;20:958-963.

35. Rabey JM, Prokhorov T, Miniovitz A, Dobronevsky E, Klein C. Effect of quetiapine in psychotic Parkinson's disease patients: a double-blind labeled study of 3 months' duration. Mov Disord 2007;22:313-318.
36. Morgante L, Epifanio A, Spina E, Zappia M, Di Rosa AE, Marconi R, et al. Quetiapine and clozapine in parkinsonian patients with dopaminergic psychosis. Clin Neuropharmacol 2004;27:153-156.

37. Broadstock M, Ballard C, Corbett A. Novel pharmaceuticals in the treatment of psychosis in Parkinson's disease. Expert Rev Clin Pharmacol 2014;7:779-786.

38. Nagata T, Shinagawa S, Tagai K, Nakayama K. A case in which mirtazapine reduced auditory hallucinations in a patient with Parkinson disease. Int Psychogeriatr 2013;25:1199-1201.

39. Tagai K, Nagata T, Shinagawa S, Tsuno N, Ozone M, Nakayama K. Mirtazapine improves visual hallucinations in Parkinson's disease: a case report. Psychogeriatrics 2013;13:103-107.

40. Nishioka K, Tanaka R, Shimura H, Hirano K, Hatano T, Miyakawa K, et al. Quantitative evaluation of electroconvulsive therapy for Parkinson's disease with refractory psychiatric symptoms. J Neural Transm (Vienna) 2014;121:1405-1410.

41. Boroojerdi B, Prager A, Muellbacher W, Cohen LG. Reduction of human visual cortex excitability using $1-\mathrm{Hz}$ transcranial magnetic stimulation. Neurology 2000;54:1529-1531.

42. Merabet LB, Kobayashi M, Barton J, Pascual-Leone A. Suppression of complex visual hallucinatory experiences by occipital transcranial magnetic stimulation: a case report. Neurocase 2003;9:436-440.

43. Burn D, Emre M, McKeith I, De Deyn PP, Aarsland D, Hsu C, et al. Effects of rivastigmine in patients with and without visual hallucinations in dementia associated with Parkinson's disease. Mov Disord 2006;21: 1899-1907.

44. Bullock R, Cameron A. Rivastigmine for the treatment of dementia and visual hallucinations associated with Parkinson's disease: a case series. Curr Med Res Opin 2002;18:258-264.

45. Cummings JL. Cholinesterase inhibitors: a new class of psychotropic compounds. Am J Psychiatry 2000;157:4-15.

46. Blom JD, Coebergh JA, Lauw R, Sommer IE. Musical hallucinations treated with acetylcholinesterase inhibitors. Front Psychiatry 2015;6:46. 